\title{
A Method of Multiple Attribute Group Decision Making Based on 2-Tuple Linguistic Dependent Maclaurin Symmetric Mean Operators
}

\author{
Min Feng ${ }^{1}$, Peide Liu ${ }^{2, *}$ and Yushui Geng ${ }^{3, *}$ \\ 1 School of Information, Qilu University of Technology, Jinan 250353, China; fengm1658@gmail.com \\ 2 School of Management Science and Engineering, Shandong University of Finance and Economics, \\ Jinan 250014, China \\ 3 Graduate School, Qilu University of Technology, Jinan 250353, China \\ * Correspondence: liupd@sdufe.edu.cn or peide.liu@gmail.com (P.L.); gys@qlu.edu.cn (Y.G.); \\ Tel.: +86-139-6405-6802 (P.L.); +86-150-6333-8888 (Y.G.)
}

Received: 3 December 2018; Accepted: 15 December 2018; Published: 1 January 2019

\begin{abstract}
Aiming at multiple attribute group decision making (MAGDM) problems, especially the attribute values of 2-tuple linguistic numbers and the interrelationships between each attribute needing to be considered, this paper proposes a new method of analysis. Firstly, we developed a few new aggregation operators, like the 2-tuple linguistic dependent weighted Maclaurin symmetric mean (2TLDWMSM) operator, the 2-tuple linguistic dependent weighted generalized Maclaurin symmetric mean (2TLDWGMSM) operator, and the 2-tuple linguistic dependent weighted geometric Maclaurin symmetric mean (2TLDWGeoMSM) operator. In the above operators, Maclaurin symmetric mean (MSM) operators can take the relationships between each attribute into account and dependent operators can mitigate the unfair parameters' impact on the overall outcome, in which those "incorrect" and "prejudiced" parameters are distributed with low weights. Next, a method used by the 2TLDWMSM, 2TLDWGMSM, and 2TLDWGeoMSM operators for MAGDM is introduced. Finally, there is an explanative example to confirm the proposed approach and explain its availability and usefulness.
\end{abstract}

Keywords: 2-tuple linguistic information; maclaurin symmetric mean; dependent operator; multiple attribute group decision making

\section{Introduction}

The problem with multiple attribute group decision making (MAGDM) is that a group of experts convey their judgements based on predefined options and choose the best one, which is a significant sector in decision theory. Owing to the uncertainties of the objects in question and the fuzziness of human thought, there are many decision-making problems in which the attributes cannot be expressed by crisp numbers, but rather by linguistic terms. For instance, when assessing a student's overall quality or driving performance, we usually use linguistic terms like "good", "medium good", "medium", "medium poor", or "poor" instead of numeric values. From this point of view, Zadeh [1] proposed a new concept of linguistic variables, which gets accomplishment in analyzing qualitative information. In addition, some methods and aggregation operators are investigated on the basis of linguistic variables for handling linguistic information, which is very helpful for processing linguistic multiple attribute decision making (MADM) problems. Yu et al. [2] developed an interactive multiple criteria decision making (MCDM) approach in which the attribute values are in intuitionistic linguistic numbers. In addition, Wang et al. [3] presented a likelihood-based TODIM (an acronym in Portuguese of interactive and multiple attribute decision making) method for dealing with multiple hesitant 
fuzzy linguistic information based on hesitation. Moreover, for purposes of simulating human perceptions of linguistic terms, Moharrer et al. [4] developed a two-phase methodology with interval type-2 fuzzy sets. In order to aggregate linguistic preference relations, Herrera et al. [5,6] developed the linguistic ordered weighted averaging (LOWA) operators. On this basis, $\mathrm{Xu}$ [7] introduced a method with linguistic preference correlations for some linguistic geometric operators. To tackle the problem of missing information during the calculation, Herrera and Martinez [8] came up with 2-tuple linguistic representation methods that describe the linguistic information recurring for 2-tuples, which overcame the limitations of missing information. After that, the 2-tuple linguistic models have solved many problems effectively in the field of MAGDM. Jiang and Fan [9] extended the traditional ordered weighted geometry (OWG) operator to a new OWG operator with 2-tuple, which enriched the analysis method of aggregating 2-tuple linguistic information. Wei [10] proposed some 2-tuple linguistic weighted operator and studied their nature. Merigo' et al. [11] put forward the induced 2-tuple linguistic operators to analyze the problem from different perspectives and have a more comprehensive understanding of the situation under consideration. Furthermore, Wang et al. [12] introduced multi-hesitant fuzzy linguistic term sets (MHFLTSs), which extend prior methods and consists of continuous and repeatable linguistic terms. Deng et al. [13] proposed the 2-tuple linguistic Pythagorean fuzzy Heronian mean (2TLPFHM) operator, which can describe the fuzzy information easily and capture interrelationships among any number of arguments.

The aggregation operator is an efficient tool for tackling MAGDM problems and it has two main functions as follows: (1) Aggregation operators can emphasize the relationship between any two attributes rather than only analyze the significance of each data or their ordered position. For example, Maclaurin [14] proposed the Maclaurin symmetric mean (MSM) operator, which could detect the correlation between multiple input arguments. After that, Detemple and Robertson [15] further developed it. Recently, the MSM has derived many significant results and received increasing attention in practical applications. Qin and Liu [16] developed some new aggregation operators that extended the MSM operator to address intuitionistic fuzzy information. At the same time, a procedure for MADM based on weighted intuitionistic fuzzy Maclaurin symmetric mean (WIFMSM) was developed. Moreover, in order to better aggregate the hesitant fuzzy information, Qin et al. [17] developed a hesitant fuzzy MSM (HFMSM) operator, which according to traditional MSM operators runs under the condition of hesitant fuzziness. In addition, neutrosophic sets are also considered. Wang et al. [18] introduced some single-valued neutrosophic linguistic MSM operators. Considering interval neutrosophic linguistic sets, some interval neutrosophic linguistic MSM operators were proposed by Geng et al. [19], which further enriched the solution to the problem of uncertain linguistic environments. In addition, Wang et al. [20] developed the interval-valued 2-tuple linguistic Pythagorean fuzzy MSM (IV2TLPFMSM) operator based on the MSM operator, generalized MSM (GMSM) operator, and dual MSM (DMSM) operator. (2) Aggregation operators can mitigate the unjust parameters' impact on the overall outcomes in which those "incorrect" and "prejudiced" parameters are distributed with low weights. Therefore, some dependent aggregation operators are investigated. For instance, $\mathrm{Xu}$ [21] proposed some dependent operators based on the uncertain ordered weighted averaging (UOWA) operator to remove the misjudgment effect on results. Wei and Zhao [22] developed several dependent ordered weighted operators with 2-tuple and Liu [23] proposed some generalized dependent operators, in which the linked weights merely rely on the aggregated input parameters. Moreover, the impact of unjust parameters on the overall outcomes in which those "wrong" and "prejudiced" parameters distributed with low weights can be relieved. In order to show the influence of the decision makers psychological factors on the group decision process, Gao and Liu [24] developed a new class of aggregation operators based on reference-dependent utility functions.

Owing to time stress, knowledge deficit, and limited experience in the field of the problem, taking the form of 2-tuple linguistic variables is very suitable for depicting vague information. Therefore, the research on the MAGDM problems based on the 2-tuple linguistic variables has great importance. Besides which, the MSM is flexible and adaptable, meaning it can detect relationships between multiple 
input parameters. The dependent aggregation operators can mitigate the impact of unjust parameters on the overall outcomes, in which those "incorrect" and "prejudiced" parameters are distributed with low weights. At present, there is limited research on the MAGDM method based on 2-tuple linguistic dependent MSM operators. MSM operators cannot handle fuzzy information in the form of 2-tuples. In addition, it is more objective to calculate weights by using dependent operators than to assign weights. If the MSM operator is used to process 2-tuple linguistic information and the dependent operator is used to calculate weight, the final decision result will be more accurate and objective. For the above reasons, this paper proposes some operators like the 2-tuple linguistic dependent weighted MSM (2TLDWMSM) operator, the 2-tuple linguistic dependent weighted generalized MSM (2TLDWGMSM) operator and the 2-tuple linguistic dependent weighted geometric MSM (2TLDWGeoMSM) operator. At the same time, a MADGM method is proposed based on above operators and display its merits by comparing with other methods.

The structure of this paper is as follows. In Section 2, some basic concepts of 2-tuple and the MSM operator are to be introduced respectively. Section 3 introduces the 2TLMSM operator and proposes the 2TLDWMSM operator, the 2TLDWGMSM operator and the 2TLDWGeoMSM operator, in which the related weights merely rely on the aggregated 2-tuple linguistic parameters and could mitigate unequitable parameters' impact on the overall outcomes in which those "wrong" and "prejudiced" ones are distributed low weights to. In Section 4 of this paper, a MAGDM method based on 2TLDWMSM operator is presented. Section 5 provides an illustrative instance to expound the advantages of the proposed method. Eventually, the conclusions and directions of further researches are proposed.

\section{Preliminaries}

\subsection{The Linguistic Set and 2-tuple}

Assume that $S\left(s_{0}, s_{1}, s_{2} \ldots s_{l-1}\right)$ is a limited strictly ordered set of discrete items and $l$ is an odd number. In actual conditions, $l$ can be equal to $3,5,7,9,11$, etc. For instance, if $l=7$, a set $S$ could be expressed:

$S=\left(s_{0}, s_{1}, s_{2}, s_{3}, s_{4}, s_{5}, s_{6}\right)=$ (extremely bad, bad, slightly bad, fair, slightly good, good, extremely good\}.

In general, for any linguistic set $S$, the following characteristics should be satisfied [25-27]:

(1) $s_{i}<s_{j}$ if and only if $i<j$;

(2) $n e g\left(s_{i}\right)=s_{l-i-1}$ is a negation operator;

(3) $\max \left(s_{i}, s_{j}\right)=s_{i}$ is a maximum operator if $i \geq j$;

(4) $\min \left(s_{i}, s_{j}\right)=s_{j}$ is a minimum operator If $i \geq j$.

As for any linguistic set $S=\left(s_{0}, s_{1}, \ldots, s_{l-1}\right)$, the correlation between $s_{i}$ and its subscript $i$ are strictly monotonically increasing $[25,27,28]$, so the definition of the function could be represented as $f: s_{i}=f(i)$. Obviously, as for subscript $i$, the function $f(i)$ is a strictly monotonically increasing function. In order to retain all the given information, the discrete linguistic label $S=\left(s_{0}, s_{1}, \ldots, s_{l-1}\right)$ is extended into a consequent linguistic label $\bar{S}=\left\{s_{a} \mid \alpha \in R\right\}$ that meets the above features.

The operational rules for the linguistic label are shown below $[27,29]$ :

$$
\begin{gathered}
\beta s_{i}=s_{\beta \times i} \beta \geq 0 \\
s_{i} \oplus s_{j}=s_{i+j} \\
s_{i} / s_{j}=s_{i / j} \\
\left(s_{i}\right)^{n}=s_{i^{n}} \\
\lambda\left(s_{i} \oplus s_{j}\right)=\lambda s_{i} \oplus \lambda s_{j} \lambda \geq 0
\end{gathered}
$$




$$
\left(\lambda_{1}+\lambda_{2}\right) s_{i}=\lambda_{1} s_{i} \oplus \lambda_{2} s_{i} \lambda_{1}, \lambda_{2} \geq 0
$$

For the sake of handling the linguistic information more easily, Herrera and Martinez [8] proposed a symbolic translation method consisting of 2-tuple. We would give the related definitions.

Definition $1([8,27,30])$. Suppose $S=\left(s_{0}, s_{1}, \ldots, s_{l-1}\right)$ is a linguistic term collection, $\beta$ is a real number in $[0, l-1]$, and it indicates the calculating result for an element in $S$, then the 2-tuple corresponding to the elements in $S$ can be get from the following function:

$$
\begin{gathered}
\Delta:[0, l-1] \rightarrow S \times[-0.5,0.5) \\
\Delta(\beta)=\left(s_{i}, \alpha\right)
\end{gathered}
$$

where $i=\operatorname{round}(\beta), \alpha=\beta-i, \alpha \in[-0.5,0.5)$, and round(.) is the usual round operation.

Definition 2 ([8,27,30]). Let $S=\left(s_{0}, s_{1}, \ldots, s_{l-1}\right)$ be a linguistic term collection, $\left(s_{i}, \alpha\right)$ be a 2-tuple, and the inverse function $\Delta^{-1}$ that converts a 2-tuple into the homologous real number $\beta \in[0, l-1]$, as follows:

$$
\begin{gathered}
\Delta^{-1}: S \times[-0.5,0.5) \rightarrow[0, l-1] \\
\Delta^{-1}\left(s_{i}, \alpha\right)=i+\alpha=\beta
\end{gathered}
$$

From this, it can be concluded that the 2-tuple corresponding to the element $s_{i}(i=0,1, \ldots l-1)$ is $\left(s_{i}, 0\right)$.

Based on above definitions, related inverse operator and comparing of 2-tuple can be easily shown as follows.

(1) The inverse operator Neg [8,27]: $\operatorname{Neg}\left(s_{i}, \alpha\right)=\Delta\left((l-1)-\left(\Delta^{-1}\left(s_{i}, \alpha\right)\right)\right)$

(2) The comparison of 2-tuple: Let $\left(s_{i}, \alpha_{1}\right)$ and $\left(s_{j}, \alpha_{2}\right)$ be any two 2-tuple, and the comparison rules are as follows $[8,27]$ :

If $i>j$ then $\left(s_{i}, \alpha_{1}\right)>\left(s_{j}, \alpha_{2}\right)$;

If $i=j$ then

(a) if $\alpha_{1}=\alpha_{2}$ then $\left(s_{i}, \alpha_{1}\right)=\left(s_{j}, \alpha_{2}\right)$;

(b) if $\alpha_{1}>\alpha_{2}$ then $\left(s_{i}, \alpha_{1}\right)>\left(s_{j}, \alpha_{2}\right)$;

(c) if $\alpha_{1}<\alpha_{2}$ then $\left(s_{i}, \alpha_{1}\right)<\left(s_{j}, \alpha_{2}\right)$.

(3) If $\left(s_{i}, \alpha_{1}\right) \geq\left(s_{j}, \alpha_{2}\right)$ then $\max \left\{\left(s_{i}, \alpha_{1}\right),\left(s_{j}, \alpha_{2}\right)\right\}=\left(s_{i}, \alpha_{1}\right)$; and if $\left(s_{i}, \alpha_{1}\right) \leq\left(s_{j}, \alpha_{2}\right)$ then $\min \left\{\left(s_{i}, \alpha_{1}\right),\left(s_{j}, \alpha_{2}\right)\right\}=\left(s_{i}, \alpha_{1}\right)$.

\subsection{Maclaurin Symmetric Mean Operator}

Definition $3([14,18])$. Let $x_{i}(i=1,2, \ldots, n)$ be the collection of the non-negative real number. An MSM operator of dimension $\mathrm{n}$ is mapping $M S M^{(m)}:\left(R^{+}\right)^{n} \rightarrow R^{+}$and it's definition is as follows:

$$
\operatorname{MSM}^{(m)}\left(x_{1}, \ldots, x_{n}\right)=\left(\frac{\sum_{1 \leq i_{1}<\ldots<i_{m} \leq n} \prod_{j=1}^{m} x_{i_{j}}}{C_{n}^{m}}\right)^{\frac{1}{m}}
$$

where $\left(i_{1}, i_{2}, \ldots, i_{m}\right)$ traverses all the $m$-tuple combination of $(1,2, \ldots, n)$ and $C_{n}^{m}=\frac{n !}{m !(n-m) !}$ is the binomial coefficient. Moreover, the meaning of $x_{i_{j}}$ is $i_{j}$ th element in a special arrangement.

Obviously, $\mathrm{MSM}^{(m)}$ operator has the following significant attributes:

(1) Idempotency. If $x_{i}=x$ for each $i, \operatorname{MSM}^{(m)}(x, x, \ldots, x)=x$; 
(2) Monotonicity. If $x_{i} \leq y_{i}$ for all $i, \operatorname{MSM}^{(m)}\left(x_{1}, x_{2}, \ldots, x_{n}\right) \leq \operatorname{MSM}^{(m)}\left(y_{1}, y_{2}, \ldots, y_{n}\right)$;

(3) Boundedness. $\min \left\{x_{1}, x_{2}, \ldots, x_{n}\right\} \leq M S M^{(m)}\left\{x_{1}, x_{2}, \ldots, x_{n}\right\} \leq \max \left\{x_{1}, x_{2}, \ldots, x_{n}\right\}$.

Definition 4 ([18]). Let $x_{i}(i=1,2, \ldots, n)$ be a collection of non-negative real numbers and $p_{1}, p_{2}, \ldots, p_{m} \geq 0$. A generalized MSM operator of dimension $n$ is mapping $\operatorname{GMSM}^{\left(m, p_{1}, p_{2}, \ldots, p_{m}\right)}:\left(R^{+}\right)^{n} \rightarrow R^{+}$and it is defined as follows:

$$
\operatorname{GMSM}^{\left(m, p_{1}, p_{2}, \ldots, p_{m}\right)}\left(x_{1}, \ldots, x_{n}\right)=\left(\frac{\sum_{1 \leq i_{1}<\ldots<i_{m}<n} \prod_{j=1}^{m} x_{i_{j}}^{p_{j}}}{C_{n}^{m}}\right)^{\frac{1}{p_{1}+p_{2}+\ldots p_{m}}}
$$

where $\left(i_{1}, i_{2}, \ldots, i_{m}\right)$ traverses all the $m$-tuple combination of $(1,2, \ldots, n)$ and $C_{n}^{m}=\frac{n !}{m !(n-m) !}$ is the binomial coefficient.

Some desirable properties of the $G M S M^{\left(m, p_{1}, p_{2}, \ldots, p_{m}\right)}$ operator are as follows:

(1) Idempotency. If $x_{i}=x$ for each $i$, and then $\operatorname{GMSM}^{\left(m, p_{1}, p_{2}, \ldots, p_{m}\right)}(x, x, \ldots, x)=x$;

(2) Monotonicity. If $x_{i} \leq y_{i}$ for all $i, G M S M^{\left(m, p_{1}, p_{2}, \ldots, p_{m}\right)}\left(x_{1}, x_{2}, \ldots, x_{n}\right) \leq$ $\operatorname{GMSM}^{\left(m, p_{1}, p_{2}, \ldots, p_{m}\right)}\left(y_{1}, y_{2}, \ldots, y_{n}\right)$;

(3) Boundedness. $\min \left\{x_{1}, x_{2}, \ldots x_{n}\right\} \leq \operatorname{GMSM}^{\left(m, p_{1}, p_{2}, \ldots, p_{m}\right)}\left(x_{1}, x_{2}, \ldots x_{n}\right) \leq \max \left\{x_{1}, x_{2}, \ldots x_{n}\right\}$

Definition 5 ([18]). Let $x_{i}(i=1,2, \ldots, n)$ be the collection of non-negative real numbers and $p_{1}, p_{2}, \ldots$, $p_{m} \geq 0$. A geometric MSM operator of dimension $n$ is mapping $G_{e 0} M S M^{\left(m, p_{1}, p_{2}, \ldots, p_{m}\right)}:\left(R^{+}\right)^{n} \rightarrow R^{+}$, and it is defined as follows:

$$
G_{e 0} M_{S M} M^{\left(m, p_{1}, p_{2}, \ldots, p_{m}\right)}\left(x_{1}, \ldots, x_{n}\right)=\frac{1}{\left(p_{1}+p_{2}+\ldots+p_{m}\right)}\left(\prod_{1 \leq i_{1}<\ldots<i_{m} \leq n}\left(p_{1} x_{i_{1}}+p_{2} x_{i_{2}}+\ldots+p_{m} x_{i_{m}}\right)\right)^{\frac{1}{C_{n}^{m}}}
$$

where $\left(i_{1}, i_{2}, \ldots, i_{m}\right)$ traverses all the m-tuple combinations of $(1,2, \ldots, n)$, and $C_{n}^{m}=\frac{n !}{m !(n-m) !}$ is the binomial coefficient.

Some desirable properties of the $G_{e 0} M S M^{\left(m, p_{1}, p_{2}, \ldots, p_{m}\right)}$ operator are as follows:

(1) Idempotency. If $x>0$, and $x_{i}=x$ for each $i$, then $G_{e o} M_{S M}^{\left(m, p_{1}, p_{2}, \ldots, p_{m}\right)}(x, x, \ldots, x)=x$;

(2) Monotonicity. If $x_{i} \leq y_{i}$ for all $i, G_{e 0} M_{S M}^{\left(m, p_{1}, p_{2}, \ldots, p_{m}\right)}\left(x_{1}, x_{2} \ldots, x_{n}\right) \leq$ $G_{e O} M_{S} M^{\left(m, p_{1}, p_{2}, \ldots, p_{m}\right)}\left(y_{1}, y_{2} \ldots, y_{n}\right)$;

(3) Boundedness. $\min \left\{x_{1}, x_{2}, \ldots x_{n}\right\} \leq G_{e o} M S M^{\left(m, p_{1}, p_{2}, \ldots, p_{m}\right)}\left(x_{1}, x_{2}, \ldots x_{n}\right) \leq \max \left\{x_{1}, x_{2}, \ldots x_{n}\right\}$.

\section{The 2-tuple Linguistic Dependent Weighted Aggregation Maclaurin Symmetric Mean Operators}

\subsection{The 2-tuple Linguistic MSM Operators}

Definition 6 ([31]). Let $x=\left\{\left(r_{1}, a_{1}\right),\left(r_{2}, a_{2}\right), \ldots,\left(r_{n}, a_{n}\right)\right\}$ be a collection of 2-tuple and let $p, q>0$. If

$$
\left.2 \operatorname{TLMSM}^{(m)}\left(\left(r_{1}, a_{1}\right),\left(r_{2}, a_{2}\right), \ldots,\left(r_{n}, a_{n}\right)\right)=\Delta\left(\left(\frac{\sum_{1 \leq i_{1}<\ldots<i_{m} \leq n} \prod_{j=1}^{m} \Delta^{-1}\left(r_{i_{j}}, a_{i_{j}}\right)}{C_{n}^{m}}\right)\right)^{\frac{1}{m}}\right)
$$

where $\left(i_{1}, i_{2}, \ldots, i_{m}\right)$ traversal all the m-tuple combination of $(1,2, \ldots, n), C_{n}^{m}=\frac{n !}{m !(n-m) !}$ is the binomial coefficient. 
Some desirable properties of the $2 T L M S M^{(m)}$ operator are as follows:

(1) Idempotency. If $\left(r_{i}, a_{i}\right)=(r, a)$, for each $i$, then $2 \operatorname{TLMSM}^{(m)}\left(\left(r_{1}, a_{1}\right),\left(r_{2}, a_{2}\right), \ldots,\left(r_{n}, a_{n}\right)\right)=(r, a)$;

(2) Monotonicity. If $\left(r_{i}, a_{i}\right) \leq\left(r_{i}^{\prime}, a_{i}^{\prime}\right)$, for all $i$, then $2 \operatorname{TLMSM}^{(m)}\left(\left(r_{1}, a_{1}\right),\left(r_{2}, a_{2}\right), \ldots,\left(r_{n}, a_{n}\right)\right) \leq$ $2 T L M S M^{(m)}\left(\left(r_{1}^{\prime}, a_{1}^{\prime}\right),\left(r_{2}^{\prime}, a_{2}^{\prime}\right), \ldots,\left(r_{n}^{\prime}, a_{n}^{\prime}\right)\right)$;

(3) Boundedness. $\min \left(r_{i}, a_{i}\right) \leq 2 \operatorname{TLMSM}^{(m)}\left(\left(r_{1}, a_{1}\right),\left(r_{2}, a_{2}\right), \ldots,\left(r_{n}, a_{n}\right)\right) \leq \max \left(r_{i}, a_{i}\right)$;

Definition 7 ([31]). Let $x=\left\{\left(r_{1}, a_{1}\right),\left(r_{2}, a_{2}\right), \ldots,\left(r_{n}, a_{n}\right)\right\}$ be a collection of 2-tuple and $m>0$, $w=\left(w_{1}, w_{2}, \ldots, w_{n}\right)^{T}$ is the weight vector of $\left(r_{i}, a_{i}\right)(i=1,2, \ldots, n)$, the 2-tuple linguistic weighted MSM operator of dimension $n$ is mapping $2 \operatorname{TLWMSM}_{w}^{(m)}:\left(R^{+}\right)^{n} \rightarrow R^{+}$, and it's definition is as follows:

$$
\operatorname{TLWMSM}_{w}^{(m)}\left(\left(r_{1}, a_{1}\right),\left(r_{2}, a_{2}\right), \ldots,\left(r_{n}, a_{n}\right)\right)=\Delta\left(\left(\frac{\sum_{1 \leq i_{1}<\ldots<i_{m} \leq n} \prod_{j=1}^{m}\left(w_{j} \Delta^{-1}\left(r_{i_{j}}, a_{i_{j}}\right)\right)}{C_{n}^{m}}\right)^{\frac{1}{m}}\right)
$$

where $w_{i}$ shows the significance degree of $\left(r_{i}, a_{i}\right)$, satisfying $w_{i}>0(i=1,2, \ldots, n)$, and $\sum_{i=1}^{n} w_{i}=1$, $\left(i_{1}, i_{2}, \ldots, i_{m}\right)$ traversal all the $m$-tuple combination of $(i=1,2, \ldots, n), C_{n}^{m}=\frac{n !}{m !(n-m) !}$ is the binomial coefficient.

Example 1. Let $x=\left\{\left(\mathrm{s}_{6}, 0\right),\left(\mathrm{s}_{2}, 0\right),\left(\mathrm{s}_{4}, 0\right),\left(\mathrm{s}_{5}, 0\right)\right\}$ be a set of 2-tuple and $S=\left\{s_{0}=\right.$ extremely bad, $s_{1}=$ bad, $s_{2}=$ slightly bad, $s_{3}=$ fair, $s_{4}=$ slightly good, $s_{5}=$ good, $s_{6}=$ extremely good $\}$, the weight vector of each 2-tuple is $\omega=(0.4,0.2,0.3,0.1)^{\mathrm{T}}$. Then the following results can be obtained:

$$
\begin{aligned}
& 2 \operatorname{LLWMSM}_{w}^{(2)}\left(\left(\mathrm{s}_{6}, 0\right),\left(\mathrm{s}_{2}, 0\right),\left(\mathrm{s}_{4}, 0\right),\left(\mathrm{s}_{5}, 0\right)\right) \\
& \left.=\Delta\left(\left(\begin{array}{l}
0.4 \times 6 \times 0.2 \times 2+0.4 \times 6 \times 0.3 \times 4+0.4 \times 6 \times 0.1 \times 5+0.2 \times 2 \times 0.3 \times 4+0.2 \times 2 \times 0.1 \times 5+ \\
0.3 \times 4 \times 0.1 \times 5
\end{array}\right)^{\frac{1}{2}}\right)\right) \\
& =\Delta(1.0263)=\left(\mathrm{s}_{1}, 0.0263\right)
\end{aligned}
$$

Definition 8. Let $x=\left\{\left(r_{1}, a_{1}\right),\left(r_{2}, a_{2}\right), \ldots,\left(r_{n}, a_{n}\right)\right\}$ be a set of 2-tuple and $m>0, p_{1}, p_{2}, \ldots, p_{m} \geq 0$, $w=\left(w_{1}, w_{2}, \ldots, w_{n}\right)^{T}$ is the weight vector of $\left(r_{i}, a_{i}\right)(i=1,2, \ldots, n)$, the 2-tuple linguistic weighted generalized MSM operator of dimension $n$ is mapping 2 TLWGMSM $M_{w}^{(m)}:\left(R^{+}\right)^{n} \rightarrow R^{+}$, and it is defined as follows:

$$
\begin{aligned}
& \text { 2TLWGMSM } M_{w}^{\left(m, p_{1}, p_{2}, \ldots, p_{m}\right)}\left(\left(r_{1}, a_{1}\right),\left(r_{2}, a_{2}\right), \ldots,\left(r_{n}, a_{n}\right)\right) \\
& \left.=\Delta\left(\left(\frac{1 \leq i_{1}<\ldots<i m \leq n j=1}{\sum_{n}^{m}\left(w_{j} \Delta^{-1}\left(r_{i}, a_{j}\right)\right)^{p_{j}}}\right)\right)^{\overline{p_{1}+p_{2}+\ldots+p_{m}}}\right)
\end{aligned}
$$

where $w_{i}$ shows the significance degree of $\left(r_{i}, a_{i}\right)$, satisfying $w_{i}>0(i=1,2, \ldots, n)$, and $\sum_{i=1}^{n} w_{i}=1$, $\left(i_{1}, i_{2}, \ldots, i_{m}\right)$ traversal all the m-tuple combination of $(i=1,2, \ldots, n), C_{n}^{m}=\frac{n !}{m !(n-m) !}$ is the binomial coefficient.

Definition 9. Let $x=\left\{\left(r_{1}, a_{1}\right),\left(r_{2}, a_{2}\right), \ldots,\left(r_{n}, a_{n}\right)\right\}$ be a set of 2-tuple and $m>0, p_{1}, p_{2}, \ldots, p_{m} \geq 0$, $w=\left(w_{1}, w_{2}, \ldots, w_{n}\right)^{T}$ is the weight vector of $\left(r_{i}, a_{i}\right)(i=1,2, \ldots, n)$, the 2-tuple linguistic weighted geometric MSM operator of dimension $n$ is mapping $2 T L W G_{e 0} M S M_{w}^{(m)}:\left(R^{+}\right)^{n} \rightarrow R^{+}$, and it is defined as follows: 


$$
\begin{aligned}
& 2 \operatorname{TLWG}_{e 0} \operatorname{MSM}^{\left(m, p_{1}, p_{2}, \ldots, p_{m}\right)}\left(x_{1}, \ldots, x_{n}\right) \\
& =\Delta\left(\frac{1}{\left(p_{1}+p_{2}+\ldots+p_{m}\right)}\left(\begin{array}{c}
\left.\prod_{1 \leq i_{1}<\ldots<i_{m} \leq n}\left(\begin{array}{c}
\left(p_{1} \Delta^{-1}\left(r_{i_{1}}, a_{i_{1}}\right)\right)^{w_{i_{1}}}+\left(p_{2} \Delta^{-1}\left(r_{i_{2}}, a_{i_{2}}\right)\right)^{w_{i_{2}}} \\
+\ldots+\left(p_{m} \Delta^{-1}\left(r_{i_{m}}, a_{i_{m}}\right)\right)^{w_{i_{n}}}
\end{array}\right)\right)^{\frac{1}{C_{n}^{m}}}
\end{array}\right)\right.
\end{aligned}
$$

where $w_{i}$ shows the significance degree of $\left(r_{i}, a_{i}\right)$, satisfying $w_{i}>0(i=1,2, \ldots, n)$, and $\sum_{i=1}^{n} w_{i}=1$, $\left(i_{1}, i_{2}, \ldots, i_{m}\right)$ traversal all the m-tuple combination of $(i=1,2, \ldots, n), C_{n}^{m}=\frac{n !}{m !(n-m) !}$ is the binomial coefficient.

\subsection{The 2-tuple Linguistic Dependent Weighted Aggregation Maclaurin Symmetric Mean Operators}

Definition 10 ([22]). Let $x=\left\{\left(r_{1}, a_{1}\right),\left(r_{2}, a_{2}\right), \ldots,\left(r_{n}, a_{n}\right)\right\}$ be a set of 2-tuples, the 2-tuple arithmetic mean can be obtained by following formula:

$$
(\bar{r}, \bar{a})=\Delta\left(\frac{1}{n} \sum_{j=1}^{n} \Delta^{-1}\left(r_{j}, a_{j}\right)\right), \bar{r} \in S, \bar{a} \in[-0.5,0.5)
$$

Definition 11 ([22]). Let $\left(r_{i}, a_{i}\right)$ and $\left(r_{j}, a_{j}\right)$ be two 2-tuple linguistic variables, and the definition of the distance between $\left(r_{i}, a_{i}\right)$ and $\left(r_{j}, a_{j}\right)$ are as follows:

$$
d\left(\left(r_{i}, a_{i}\right),\left(r_{j}, a_{j}\right)\right)=\frac{\left|\Delta^{-1}\left(r_{i}, a_{i}\right)-\Delta^{-1}\left(r_{j}, a_{j}\right)\right|}{l}
$$

Definition 12 ([22,23]). Let $x=\left\{\left(r_{1}, a_{1}\right),\left(r_{2}, a_{2}\right), \ldots,\left(r_{n}, a_{n}\right)\right\}$ be a collection of 2-tuples, and the arithmetic mean of 2-tuples is represented by $(\bar{r}, \bar{a})$, then we would obtain the degree of similarity between the jth largest 2-tuples linguistic variables $\left(r_{j}, a_{j}\right)$ and the mean $(\bar{r}, \bar{a})$, as follows.

$$
\operatorname{sim}\left(\left(r_{\pi(j)}, a_{\pi(\mathrm{j})}\right),(\bar{r}, \bar{a})\right)=1-\frac{d\left(\left(r_{\pi(j)}, a_{\pi(\mathrm{j})}\right),(\bar{r}, \bar{a})\right)}{\left.\sum_{j=1}^{n} d\left(\left(r_{\pi(j)}\right) a_{\pi(\mathrm{j})}\right),(\bar{r}, \bar{a})\right)}
$$

where $(\pi(1), \pi(2), \ldots, \pi(n))$ is a permutation of $(1,2, \ldots, n)$, such that $\left(r_{\pi(j-1)}, a_{\pi(j-1)}\right) \geq\left(r_{\pi(j)}, a_{\pi(j)}\right)$ for all $j=2, \ldots, n$.

In the cases of the real-life decision making, $\mathrm{n}$ different individuals would provide $\mathrm{n}$ preference values in the form of the 2-tuple $\left(r_{1}, a_{1}\right),\left(r_{2}, a_{2}\right), \ldots,\left(r_{n}, a_{n}\right)$. There is no doubt that assigning different weights will affect the final decision of the group [32]. However, for some object that decision-makers prefer or hate, some decision-making experts can assign too good or too bad preference values. Under the circumstances, "false" or "biased" opinions would be assigned lower weight. In other words, the greater the weight of the preference value (argument), the closer it is to the intermediate value [23]. So, based on (18), the weights could be defined as follows:

$$
w_{j}=\frac{\operatorname{sim}\left(\left(r_{\pi(j)}, a_{\pi(j)}\right),(\bar{r}, \bar{a})\right)}{\sum_{j=1}^{n} \operatorname{sim}\left(\left(r_{\pi(j)}, a_{\pi(j)}\right),(\bar{r}, \bar{a})\right)}, j=1,2, \ldots, n
$$

where $w_{j} \geq 0, j=1,2, \ldots, n$ and $\sum_{j=1}^{n} w_{j}=1$.

Particularly, if $\left(r_{\pi(j)}, a_{\pi(\mathrm{j})}\right)=(\bar{r}, \bar{a})$, for all $j=1,2, \ldots, n$, then by (19), $w_{j}=\frac{1}{n}$ can be obtained for all $j=1,2, \ldots, n$. In addition, the following results can be obtained:

(1) Let $x=\left\{\left(r_{1}, a_{1}\right),\left(r_{2}, a_{2}\right), \ldots,\left(r_{n}, a_{n}\right)\right\}$ be a collection of 2-tuples, and let $(\bar{r}, \bar{a})$ be the arithmetic mean of 2-tuples, $(\pi(1), \pi(2), \ldots, \pi(n))$ is a permutation of $(1,2, \ldots, n)$, such 
that $\left(r_{\pi(j-1)}, a_{\pi(j-1)}\right) \geq\left(r_{\pi(j)}, a_{\pi(j)}\right)$ for all $j=2, \ldots, n$. If $\operatorname{sim}\left(\left(r_{\pi(i)}, a_{\pi(i)}\right),(\bar{r}, \bar{a})\right) \geq$ $\operatorname{sim}\left(\left(r_{\pi(j)}, a_{\pi(\mathrm{j})}\right),(\bar{r}, \bar{a})\right)$, then $w_{i} \geq w_{j}$.

Definition 13. Let $x=\left\{\left(r_{1}, a_{1}\right),\left(r_{2}, a_{2}\right), \ldots,\left(r_{n}, a_{n}\right)\right\}$ be a collection of 2-tuple and $m>0$, the 2-tuple linguistic dependent weighted MSM operator of dimension $n$ is mapping $2 \operatorname{TLDWMSM}_{w}^{(m)}:\left(R^{+}\right)^{n} \rightarrow R^{+}$, and it is defined as follows:

$$
2 \operatorname{TLDWMSM}_{w}^{(m)}\left(\left(r_{1}, a_{1}\right),\left(r_{2}, a_{2}\right), \ldots,\left(r_{n}, a_{n}\right)\right)=\Delta\left(\left(\frac{\sum_{1 \leq i_{1}<\ldots<i_{m} \leq n} \prod_{j=1}^{m}\left(w_{i_{j}} \Delta^{-1}\left(r_{i_{j}}, a_{i_{j}}\right)\right)}{C_{n}^{m}}\right)^{\frac{1}{m}}\right)
$$

where $w=\left(w_{1}, w_{2}, \ldots, w_{n}\right)^{T}$ is a weight vector which is defined by formula $(19) ;(\pi(1), \pi(2), \ldots, \pi(n))$ is a permutation of $(1,2, \ldots, n)$.

By formula (19), we can describe formula (20) as follows.

$$
\begin{aligned}
& \text { 2TLDWMSM }{ }^{(m)}\left(\left(r_{1}, a_{1}\right),\left(r_{2}, a_{2}\right), \ldots,\left(r_{n}, a_{n}\right)\right) \\
& =\Delta\left(\left(\frac{\sum_{1 \leq i_{1}<\ldots<i_{m} \leq n} \prod_{j=1}^{m}\left(\frac{\operatorname{sim}\left(\left(r_{i_{j}}, a_{i j}\right),(\bar{r}, \bar{a})\right) \Delta^{-1}\left(r_{i_{j}, a_{i_{j}}}\right)}{\sum_{j=1}^{n} \operatorname{sim}\left(\left(r_{i_{j},}, a_{i j}\right),(\bar{p}, \bar{a})\right)}\right)}{C_{n}^{m}}\right)\right)
\end{aligned}
$$

Example 2. Let $x=\left\{\left(\mathrm{s}_{6}, 0\right),\left(\mathrm{s}_{2}, 0\right),\left(\mathrm{s}_{4}, 0\right),\left(\mathrm{s}_{5}, 0\right)\right\}$ be a set of 2-tuple and $S=\left\{s_{0}=\right.$ very bad, $s_{1}=$ bad, $s_{2}=$ slightly bad, $s_{3}=$ fair, $s_{4}=$ slightly good, $s_{5}=$ good, $s_{6}=$ very good $\}$, then the following results can be obtained:

$$
\begin{aligned}
& (\bar{r}, \bar{a})=\Delta\left(\frac{1}{4} \sum_{j=1}^{4} \Delta^{-1}\left(r_{j}, a_{j}\right)\right)=\frac{6+2+4+5}{4}=\Delta(4.25)=\left(s_{4}, 0.25\right) \\
& d\left(\left(s_{6}, 0\right),\left(s_{4}, 0.25\right)\right)=\frac{|6-4.25|}{7}=0.25, d\left(\left(s_{2}, 0\right),\left(s_{4}, 0.25\right)\right)=\frac{|2-4.25|}{7}=0.3214 \text {, } \\
& d\left(\left(s_{4}, 0\right),\left(s_{4}, 0.25\right)\right)=\frac{|4-4.25|}{7}=0.0357, d\left(\left(s_{5}, 0\right),\left(s_{4}, 0.25\right)\right)=\frac{|5-4.25|}{7}=0.1071 \\
& \operatorname{sim}\left(\left(s_{6}, 0\right),\left(s_{4}, 0.25\right)\right)=1-\frac{0.25}{0.25+0.3214+0.0357+0.1071}=0.65 \text {, } \\
& \operatorname{sim}\left(\left(s_{2}, 0\right),\left(s_{4}, 0.25\right)\right)=1-\frac{0.3214}{0.25+0.3214+0.0357+0.1071}=0.55, \\
& \operatorname{sim}\left(\left(s_{4}, 0\right),\left(s_{4}, 0.25\right)\right)=1-\frac{0.0357}{0.25+0.3214+0.0357+0.1071}=0.95, \\
& \operatorname{sim}\left(\left(s_{5}, 0\right),\left(s_{4}, 0.25\right)\right)=1-\frac{0.1071}{0.25+0.3214+0.0357+0.1071}=0.85 \text {, } \\
& w_{1}=\frac{0.65}{3}=0.2167, w_{2}=\frac{0.55}{3}=0.1833, w_{3}=\frac{0.95}{3}=0.3167, w_{4}=\frac{0.85}{3}=0.2833 \text {, } \\
& 2 T L D W M S M_{w}^{(2)}\left(\left(\mathrm{s}_{6}, 0\right),\left(\mathrm{s}_{2}, 0\right),\left(\mathrm{s}_{4}, 0\right),\left(\mathrm{s}_{5}, 0\right)\right) \\
& =\Delta\left(\left(\begin{array}{c}
0.2167 \times 6 \times 0.1833 \times 2+0.2167 \times 6 \times 0.3167 \times 4+0.2167 \times 6 \times 0.2833 \times 5+ \\
0.1833 \times 2 \times 0.3167 \times 4+0.1833 \times 2 \times 0.2833 \times 5+0.3167 \times 4 \times 0.2833 \times 5 \\
C_{4}^{2}
\end{array}\right)\right. \\
& =\Delta(1.1239)=\left(\mathrm{s}_{1}, 0.1239\right)
\end{aligned}
$$

There is a desirable property of the $2 \operatorname{TLDWMSM}{ }^{(m)}$ operator as follows: 
(1) Commutativity. Let $\left\{\left(r_{1}, a_{1}\right)^{\prime},\left(r_{2}, a_{2}\right)^{\prime}, \ldots,\left(r_{n}, a_{n}\right)^{\prime}\right\}$ be any premutation of $\left\{\left(r_{1}, a_{1}\right),\left(r_{2}, a_{2}\right), \ldots,\left(r_{n}, a_{n}\right)\right\}$, then $2 \operatorname{TLDWMSM}^{(m)}\left(\left(r_{1}, a_{1}\right)^{\prime},\left(r_{2}, a_{2}\right)^{\prime}, \ldots,\left(r_{n}, a_{n}\right)^{\prime}\right)=$ $2 T L D W M S M^{(m)}\left(\left(r_{1}, a_{1}\right),\left(r_{2}, a_{2}\right), \ldots,\left(r_{n}, a_{n}\right)\right)$

Proof. Let

$$
\begin{aligned}
& \left.2 \operatorname{TLDWMSM}^{(m)}\left(\left(r_{1}, a_{1}\right),\left(r_{2}, a_{2}\right), \ldots,\left(r_{n}, a_{n}\right)\right)=\Delta\left(\left(\frac{\sum_{1 \leq i_{1}<\ldots<i_{m} \leq n} \prod_{j=1}^{m}\left(\frac{\operatorname{sim}\left(\left(r_{i_{j}}, a_{i j}\right),(\bar{r}, \bar{a})\right) \Delta^{-1}\left(r_{i_{i}, a_{i j}}\right)}{\sum_{j=1}^{n} \operatorname{sim}\left(\left(r_{i_{j}}, a_{i_{j}}\right),(\bar{r}, \bar{a})\right)}\right)}{C_{n}^{m}}\right)\right)^{\frac{1}{m}}\right) \\
& \left.2 \operatorname{TLDWMSM}^{(m)}\left(\left(r_{1}, a_{1}\right)^{\prime},\left(r_{2}, a_{2}\right)^{\prime}, \ldots,\left(r_{n}, a_{n}\right)^{\prime}\right)=\Delta\left(\left(\frac{\sum_{1 \leq i_{1}<\ldots<i_{m} \leq n} \prod_{j=1}^{m}\left(\frac{\operatorname{sim}\left(\left(r_{i j}, a_{j}\right),(\bar{r}, \bar{a})\right) \Delta^{-1}\left(r_{i,}, a_{i j}\right)}{\sum_{j=1}^{n} \operatorname{sim}\left(\left(r_{i_{j}}, a_{i j}\right),(\bar{r}, \bar{a})\right)}\right)}{C_{n}^{m}}\right)\right)^{\frac{1}{m}}\right)
\end{aligned}
$$

Since $\left\{\left(r_{1}, a_{1}\right)^{\prime},\left(r_{2}, a_{2}\right)^{\prime}, \ldots,\left(r_{n}, a_{n}\right)^{\prime}\right\}$ is any premutation of $\left\{\left(r_{1}, a_{1}\right),\left(r_{2}, a_{2}\right), \ldots,\left(r_{n}, a_{n}\right)\right\}$, we can get $\operatorname{sim}\left(\left(r_{i_{j}}, a_{i_{j}}\right)^{\prime},(\bar{r}, \bar{a})\right) \Delta^{-1}\left(r_{i_{j}}, a_{i_{j}}\right)^{\prime}=\operatorname{sim}\left(\left(r_{i_{j}}, a_{i_{j}}\right),(\bar{r}, \bar{a})\right) \Delta^{-1}\left(r_{i_{j}}, a_{i_{j}}\right)$ and

$$
\operatorname{sim}\left(\left(r_{i_{j}}, a_{i_{j}}\right)^{\prime},(\bar{r}, \bar{a})\right)=\operatorname{sim}\left(\left(r_{i_{j}}, a_{i_{j}}\right),(\bar{r}, \bar{a})\right)
$$

Therefore, $2 \operatorname{TLDWMSM}^{(m)}\left(\left(r_{1}, a_{1}\right)^{\prime},\left(r_{2}, a_{2}\right)^{\prime}, \ldots,\left(r_{n}, a_{n}\right)^{\prime}\right)=2 \operatorname{TLDWMSM}^{(m)}$ $\left(\left(r_{1}, a_{1}\right),\left(r_{2}, a_{2}\right), \ldots,\left(r_{n}, a_{n}\right)\right)$.

Definition 14. Let $x=\left\{\left(r_{1}, a_{1}\right),\left(r_{2}, a_{2}\right), \ldots,\left(r_{n}, a_{n}\right)\right\}$ be a collection of 2-tuple and $m>0, p_{1}, p_{2}, \ldots$, $p_{m} \geq 0$, the 2-tuple linguistic dependent weighted generalized MSM operator of dimension $\mathrm{n}$ is mapping 2TLDWGMSM $M_{w}^{(m)}:\left(R^{+}\right)^{n} \rightarrow R^{+}$, and the definition is as follows:

$$
\begin{aligned}
& 2 \operatorname{TLDWGMSM} M_{w}^{\left(m, p_{1}, p_{2}, \ldots, p_{n}\right)}\left(\left(r_{1}, a_{1}\right),\left(r_{2}, a_{2}\right), \ldots,\left(r_{n}, a_{n}\right)\right) \\
& =\Delta\left(\left(\frac{\sum_{1 \leq i_{1}<\ldots<i_{m} \leq n} \prod_{j=1}^{m}\left(w_{i_{j}} \Delta^{-1}\left(r_{i_{j}}, a_{i_{j}}\right)\right)^{p_{j}}}{C_{n}^{m}}\right)^{\overline{p_{1}+p_{2}+\ldots+p_{m}}}\right)
\end{aligned}
$$

where $w=\left(w_{1}, w_{2}, \ldots, w_{n}\right)^{T}$ is a weight vector which is defined by formula $(19) ;(\pi(1), \pi(2), \ldots, \pi(n))$ is a permutation of $(1,2, \ldots, n)$.

By formula (19), we can describe formula (22) as follows:

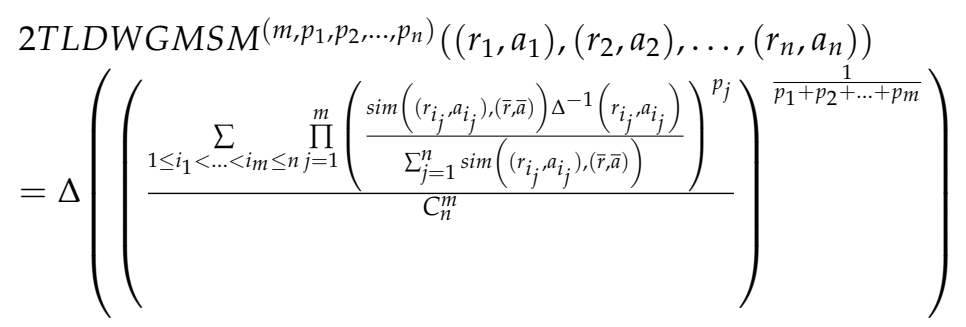

There is a desirable property of the $2 T L D W G M S M^{(m)}$ operator as follows:

(1) Commutativity. Let $\left\{\left(r_{1}, a_{1}\right)^{\prime},\left(r_{2}, a_{2}\right)^{\prime}, \ldots,\left(r_{n}, a_{n}\right)^{\prime}\right\}$ be any premutation of $\left\{\left(r_{1}, a_{1}\right),\left(r_{2}, a_{2}\right), \ldots,\left(r_{n}, a_{n}\right)\right\}$, then 2TLDWGMSM ${ }^{(m)}\left(\left(r_{1}, a_{1}\right)^{\prime},\left(r_{2}, a_{2}\right)^{\prime}, \ldots,\left(r_{n}, a_{n}\right)^{\prime}\right)=$ $2 \operatorname{TLDWGMSM}{ }^{(m)}\left(\left(r_{1}, a_{1}\right),\left(r_{2}, a_{2}\right), \ldots,\left(r_{n}, a_{n}\right)\right)$. 
Because this property is analogous to the property of $2 \operatorname{TLDWMSM}{ }^{(m)}$, the proof is omitted here.

Definition 15. Let $x=\left\{\left(r_{1}, a_{1}\right),\left(r_{2}, a_{2}\right), \ldots,\left(r_{n}, a_{n}\right)\right\}$ be a collection of 2 -tuple and $m>0, p_{1}, p_{2}, \ldots$, $p_{m} \geq 0$, the 2-tuple linguistic dependent weighted geometric Maclaurin symmetric mean operator of dimension $n$ is mapping $2 T L D W G_{e o} M S M_{w}^{(m)}:\left(R^{+}\right)^{n} \rightarrow R^{+}$, and it is defined as follows:

$$
\begin{aligned}
& 2 \operatorname{TLDWG}_{e 0} \operatorname{MSM}_{w}\left(m, p_{1}, p_{2}, \ldots, p_{m}\right)\left(x_{1}, \ldots, x_{n}\right) \\
& =\Delta\left(\frac{1}{\left(p_{1}+p_{2}+\ldots+p_{m}\right)}\left(\begin{array}{c}
\left.\left.\prod_{1 \leq i_{1}<\ldots<i_{m} \leq n}\left(\begin{array}{l}
\left(p_{1} \Delta^{-1}\left(r_{i_{1}}, a_{i_{1}}\right)\right)^{w_{i_{1}}}+\left(p_{2} \Delta^{-1}\left(r_{i_{2}}, a_{i_{2}}\right)\right)^{w_{i_{2}}} \\
+\ldots+\left(p_{m} \Delta^{-1}\left(r_{i_{m}}, a_{i_{m}}\right)\right)^{w_{i_{n}}}
\end{array}\right)\right)^{\frac{1}{C_{n}^{m}}}\right)
\end{array}\right)\right.
\end{aligned}
$$

where $w=\left(w_{1}, w_{2}, \ldots, w_{n}\right)^{T}$ is a weight vector which is defined by formula $(19) ;(\pi(1), \pi(2), \ldots, \pi(n))$ is a permutation of $(1,2, \ldots, n)$.

By formula (19), we can describe formula (24) as follows:

$$
\begin{aligned}
& 2 T L D W G_{e o} \operatorname{MSM}_{w}\left(m, p_{1}, p_{2}, \ldots, p_{m}\right)\left(x_{1}, \ldots, x_{n}\right)
\end{aligned}
$$

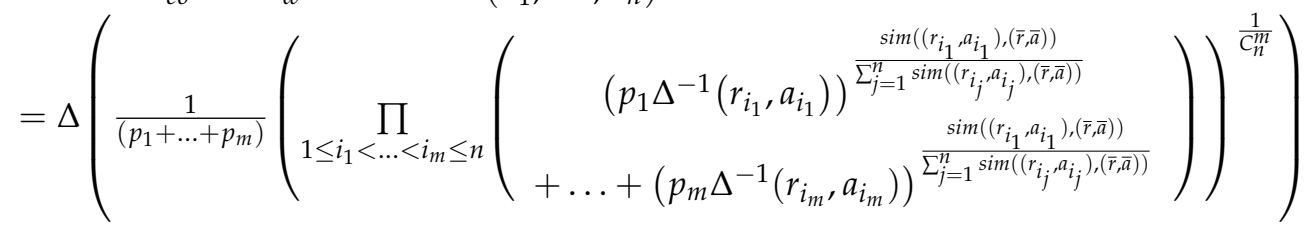

There is a desirable property of the $2 T L D W G_{e o} M S M^{(m)}$ operator as follows:

(1) Commutativity. Let $\left\{\left(r_{1}, a_{1}\right)^{\prime},\left(r_{2}, a_{2}\right)^{\prime}, \ldots,\left(r_{n}, a_{n}\right)^{\prime}\right\}$ be any premutation of $\left\{\left(r_{1}, a_{1}\right),\left(r_{2}, a_{2}\right), \ldots,\left(r_{n}, a_{n}\right)\right\}$, then $2 T L D W G_{e o} M S M^{(m)}\left(\left(r_{1}, a_{1}\right)^{\prime},\left(r_{2}, a_{2}\right)^{\prime}, \ldots,\left(r_{n}, a_{n}\right)^{\prime}\right)=$ $2 T L D W G_{e o} M_{S M}{ }^{(m)}\left(\left(r_{1}, a_{1}\right),\left(r_{2}, a_{2}\right), \ldots,\left(r_{n}, a_{n}\right)\right)$. Because this property is analogous to the property of $2 T L D W M S M^{(m)}$, the proof is omitted here.

\section{MAGDM Based on 2TLDWMSM Operator or 2TLDWGMSM Operator or 2TLDWGeoMSM Operator}

In this section, we would introduce methods based on 2TLDWMSM operator, 2TLDWGMSM operator and 2TLDWGeoMSM operator, which can deal with the MAGDM problems, in which the weights are in real numbers and the attribute preference values take the form of 2-tuple linguistic variables.

Let $A=\left\{A_{1}, A_{2}, \ldots, A_{m}\right\}$ be a discrete set of alternatives, $C=\left\{C_{1}, C_{2}, \ldots, C_{n}\right\}$ be the set of attributes, $D=\left\{D_{1}, D_{2}, \ldots, D_{p}\right\}$ be the set of decision makers and $\omega=\left\{\omega_{1}, \omega_{2}, \ldots, \omega_{n}\right\}$ be the weighting vector of the attributes $C_{j}(j=1,2, \ldots, n)$, where $\omega_{j} \in[0,1], \sum_{j=1}^{n} \omega_{j}=1$. Decision maker $D_{k}$ would provide an attribute value $x_{i_{j}}^{k}$ for the alternative $A_{i} \in A$ about the attribute $C_{j} \in C$, and it is in the form of linguistic variable $x_{i_{j}}^{k} \in S$. All attribute values $x_{i_{j}}^{k}$ constitute the decision matrix $X^{k}=\left[x_{i_{j}}^{k}\right]_{m \times n}$. Finally, sorting the options.

Next, we employ the 2TLDWMSM, the 2TLDWGMSM and the 2TLDWGeoMSM operator to handle MAGDM problems in which the attribute values are in 2-tuple.

The main steps of the method are shown below:

Step 1. Normalization

In general, MADM problem has two attribute types: cost-type (we hope that the value is as small as possible.) and benefit-type (we hope that the value is as large as possible.). For the sake 
of maintaining consistency of the types, we need to normalize the matrix. $X^{k}=\left[x_{i_{j}}^{k}\right]_{m \times n}$ will be converted into the matrix $R^{k}=\left[r_{i_{j}}^{k}\right]_{m \times n^{\prime}}$ where

(1) If the type of $C_{j}$ is beneficial: $r_{i_{j}}^{k}=x_{i_{j}}^{k}$

(2) If the type of $C_{j}$ is cost: $r_{i_{j}}^{k}=N e g\left(x_{i_{j}}^{k}\right)$

Step 2. Converting initial decision matrix $R^{k}=\left[r_{i_{j}}^{k}\right]_{m \times n}$ into $R=\left[\left(r_{i^{\prime}}^{k}, 0\right)\right]_{m \times n}$.

Step 3. Aggregating all of the decision matrixes $R=\left[\left(r_{i_{j}}^{k}, 0\right)\right]_{m \times n}(k=1,2, \ldots, p)$ into a decision matrix $R=\left[\left(r_{i_{j}}, a_{i_{j}}\right)\right]_{m \times n}$ using 2TLDWMSM operator or 2TLDWGMSM operator or 2TLDWGeoMSM operator.

Step 4. Calculating each alternative's comprehensive evaluation value $\left(r_{i}, a_{i}\right)$ by the 2TLDWMSM operator or 2TLDWGMSM operator or 2TLDWGeoMSM operator.

Step 5. Ranking the 2-tuple $\left(r_{i}, a_{i}\right)(i=1,2, \ldots, m)$ in the light of the comparing of 2-tuple in Section 2.1.

Step 6. Sorting alternatives $A=\left\{A_{1}, A_{2}, \ldots, A_{m}\right\}$ and select the best choice with the highest performance value.

Step 7. End.

\section{Illustrative Example}

\subsection{Data and Backdrop}

In this section, we adapted an instance from [33] to calculate and compare the methods proposed in this paper. Firstly, we would introduce the backdrop of the MAGDM: An investment company intends to have an investment in an industry to get the best benefit. There are four options: (1) $A_{1}$ represents an automotive industry; (2) $A_{2}$ represents a food industry; (3) $A_{3}$ represents a computer industry; (4) $A_{4}$ represents an arms industry. The investment company will consider the following three attributes when making decisions: (1) $C_{1}$ represents the risk index; (2) $C_{2}$ represents the growth index; (3) $C_{3}$ represents the social-political impact index. The three decision makers evaluated the four choices $A_{i}(i=1,2,3,4)$ based on the above three attributes $C_{i}(i=1,2,3)$. The decision matrices $X^{k}=\left[x_{i_{j}}^{k}\right]_{4 \times 3}$ are listed in Tables $1-3$, where $x_{i_{j}} \in S, S=\left(s_{0}, s_{1}, s_{2}, s_{3}, s_{4}, s_{5}, s_{6}, s_{7}, s_{8}\right)=$ (particularly bad, very bad, bad, slightly bad, fair, slightly good, good, very good, extremely good).

Table 1. The attribute values evaluated by $\mathrm{D} 1$.

\begin{tabular}{cccc}
\hline Options $\backslash$ Attributes & $C_{1}$ & $C_{2}$ & $C_{3}$ \\
\hline$A_{1}$ & $s_{5}$ & $s_{7}$ & $s_{7}$ \\
$A_{2}$ & $s_{6}$ & $s_{4}$ & $s_{5}$ \\
$A_{3}$ & $s_{3}$ & $s_{4}$ & $s_{6}$ \\
$A_{4}$ & $s_{6}$ & $s_{4}$ & $s_{6}$ \\
\hline
\end{tabular}

Table 2. The attribute values evaluated by D2.

\begin{tabular}{cccc}
\hline Options $\backslash$ Attributes & $C_{1}$ & $C_{2}$ & $C_{3}$ \\
\hline$A_{1}$ & $s_{4}$ & $s_{5}$ & $s_{4}$ \\
$A_{2}$ & $s_{7}$ & $s_{6}$ & $s_{5}$ \\
$A_{3}$ & $s_{4}$ & $s_{5}$ & $s_{6}$ \\
$A_{4}$ & $s_{5}$ & $s_{4}$ & $s_{5}$ \\
\hline
\end{tabular}


Table 3. The attribute values evaluated by D3.

\begin{tabular}{cccc}
\hline Options $\backslash$ Attributes & $C_{1}$ & $C_{2}$ & $C_{3}$ \\
\hline$A_{1}$ & $s_{3}$ & $s_{5}$ & $s_{4}$ \\
$A_{2}$ & $s_{7}$ & $s_{6}$ & $s_{5}$ \\
$A_{3}$ & $s_{5}$ & $s_{4}$ & $s_{7}$ \\
$A_{4}$ & $s_{7}$ & $s_{6}$ & $s_{5}$ \\
\hline
\end{tabular}

\subsection{The Method Based on the 2TLDWMSM Operator}

In general, we set $m=2$, according to Section 4 and the procedures of the method are as follows:

Step 1. Normalizing the matrices

Since values of each attribute are benefit-type, we have $R^{k}=\left[r_{i_{j}}^{k}\right]_{m \times n}=\left[x_{i_{j}}^{k}\right]_{m \times n}$.

Step 2. Converting initial linguistic information decision matrices $R^{k}=\left[r_{i_{j}}^{k}\right]_{m \times n}$ given in Tables 1-3 into matrices $R^{k}=\left[\left(r_{i_{j}}^{k}, 0\right)\right]_{m \times n}$ which are given in Tables 4-6.

Table 4. 2-tuple linguistic decision matrix R1.

\begin{tabular}{cccc}
\hline Options $\backslash$ Attributes & $\boldsymbol{C}_{1}$ & $\boldsymbol{C}_{2}$ & $\boldsymbol{C}_{3}$ \\
\hline$A_{1}$ & $\left(s_{5}, 0\right)$ & $\left(s_{7}, 0\right)$ & $\left(s_{7}, 0\right)$ \\
$A_{2}$ & $\left(s_{6}, 0\right)$ & $\left(s_{4}, 0\right)$ & $\left(s_{5}, 0\right)$ \\
$A_{3}$ & $\left(s_{3}, 0\right)$ & $\left(s_{4}, 0\right)$ & $\left(s_{6}, 0\right)$ \\
$A_{4}$ & $\left(s_{6}, 0\right)$ & $\left(s_{4}, 0\right)$ & $\left(s_{6}, 0\right)$ \\
\hline
\end{tabular}

Table 5. 2-tuple linguistic decision matrix R2.

\begin{tabular}{cccc}
\hline Options $\backslash$ Attributes & $\boldsymbol{C}_{1}$ & $\boldsymbol{C}_{2}$ & $\boldsymbol{C}_{3}$ \\
\hline$A_{1}$ & $\left(s_{4}, 0\right)$ & $\left(s_{5}, 0\right)$ & $\left(s_{4}, 0\right)$ \\
$A_{2}$ & $\left(s_{7}, 0\right)$ & $\left(s_{6}, 0\right)$ & $\left(s_{5}, 0\right)$ \\
$A_{3}$ & $\left(s_{4}, 0\right)$ & $\left(s_{5}, 0\right)$ & $\left(s_{6}, 0\right)$ \\
$A_{4}$ & $\left(s_{5}, 0\right)$ & $\left(s_{4}, 0\right)$ & $\left(s_{5}, 0\right)$ \\
\hline
\end{tabular}

Table 6. 2-tuple linguistic decision matrix R3.

\begin{tabular}{cccc}
\hline Options $\backslash$ Attributes & $\boldsymbol{C}_{1}$ & $\boldsymbol{C}_{2}$ & $\boldsymbol{C}_{3}$ \\
\hline$A_{1}$ & $\left(s_{3}, 0\right)$ & $\left(s_{5}, 0\right)$ & $\left(s_{4}, 0\right)$ \\
$A_{2}$ & $\left(s_{7}, 0\right)$ & $\left(s_{6}, 0\right)$ & $\left(s_{5}, 0\right)$ \\
$A_{3}$ & $\left(s_{5}, 0\right)$ & $\left(s_{4}, 0\right)$ & $\left(s_{7}, 0\right)$ \\
$A_{4}$ & $\left(s_{7}, 0\right)$ & $\left(s_{6}, 0\right)$ & $\left(s_{5}, 0\right)$ \\
\hline
\end{tabular}

Step 3. Aggregating all the decision matrixes $R^{k}=\left[\left(r_{i_{j}}^{k}, 0\right)\right]_{4 \times 3}(k=1,2,3)$ obtained in step 2 to a decision matrix $R=\left[\left(r_{i_{j}}, a_{i_{j}}\right)\right]_{4 \times 3}$ by 2TLDWMSM, then following matrix can be obtained:

$$
R\left[\begin{array}{ccc}
\left(s_{1}, 0.2829\right) & \left(s_{2},-0.1671\right) & \left(s_{2},-0.4189\right) \\
\left(s_{2}, 0.2185\right) & \left(s_{2},-0.2146\right) & \left(s_{2},-0.3333\right) \\
\left(s_{1}, 0.2829\right) & \left(s_{1}, 0.4142\right) & \left(s_{2}, 0.0767\right) \\
\left(s_{2},-0.0689\right) & \left(s_{1}, 0.5\right) & \left(s_{2},-0.2545\right)
\end{array}\right]
$$


Step 4. Calculating each alternative's comprehensive evaluation value $r_{i}=\left(r_{i}, a_{i}\right)$ by 2TLDWMSM, we would get following result:

$$
r_{1}=\left(s_{1},-0.4923\right), r_{2}=\left(s_{1},-0.3854\right), r_{3}=\left(s_{1},-0.4899\right), r_{4}=\left(s_{1},-0.4378\right)
$$

Step 5. Ranking the 2-tuple $r_{i}=\left(r_{i}, a_{i}\right)(i=1,2,3,4)$, the following sorted result can be obtained:

$$
r_{2}>r_{4}>r_{3}>r_{1}
$$

Step 6. Ranking all the alternatives $A=\left\{A_{1}, A_{2}, A_{3}, A_{4}\right\}$ in conformity to $r_{i}$, the sorted results are shown below:

$$
A_{2}>A_{4}>A_{3}>A_{1}
$$

Thus, the best one is $A_{2}$.

\subsection{The Method Based on the 2TLDWGMSM Operator}

When $m=2, p_{1}=1, p_{2}=2$ according to Section 4 , the procedures of the method are as follows:

Step 1. Normalizing the matrices

Since values of each attribute are benefit-type, we have $R^{k}=\left[r_{i_{j}}^{k}\right]_{m \times n}=\left[x_{i_{j}}^{k}\right]_{m \times n}$.

Step 2. Converting initial linguistic information decision matrices $R^{k}=\left[r_{i_{j}}^{k}\right]_{m \times n}$ given in Tables 1-3 into matrices $R^{k}=\left[\left(r_{i_{j}}^{k}, 0\right)\right]_{m \times n}$ which are displayed in Tables 4-6.

Step 3. Aggregating all decision matrixes $R^{k}=\left[\left(r_{i_{j}}^{k}, 0\right)\right]_{4 \times 3}(k=1,2,3)$ obtained in step 2 to the decision matrix $R=\left[\left(r_{i^{\prime}}, a_{i_{j}}\right)\right]_{4 \times 3}$ by 2TLDWGMSM, then following matrix can be obtained:

$$
R\left[\begin{array}{ccc}
\left(s_{1}, 0.3448\right) & \left(s_{2},-0.1667\right) & \left(s_{2},-0.4167\right) \\
\left(s_{2}, 0.2535\right) & \left(s_{2},-0.1595\right) & \left(s_{2},-0.3333\right) \\
\left(s_{1}, 0.3154\right) & \left(s_{1}, 0.4168\right) & \left(s_{2}, 0.0837\right) \\
\left(s_{2},-0.0074\right) & \left(s_{1}, 0.5\right) & \left(s_{2},-0.2498\right)
\end{array}\right]
$$

Step 4. Calculating each alternative's comprehensive evaluation value $r_{i}=\left(r_{i}, a_{i}\right)$ by 2TLDWGMSM, we would get following result:

$$
r_{1}=\left(s_{1},-0.4702\right), r_{2}=\left(s_{1},-0.3701\right), r_{3}=\left(s_{1},-0.484\right), r_{4}=\left(s_{1},-0.4223\right)
$$

Step 5. Ranking the 2-tuple $r_{i}=\left(r_{i}, a_{i}\right)(i=1,2,3,4)$, the following sorted result can be obtained:

$$
r_{2}>r_{4}>r_{1}>r_{3}
$$

Step 6. Ranking all the alternatives $A=\left\{A_{1}, A_{2}, A_{3}, A_{4}\right\}$ in conformity to $r_{i}$, the sorted results are shown below:

$$
A_{2}>A_{4}>A_{3}>A_{1}
$$

Thus, the best one is $A_{2}$.

\subsection{The Method Based on the 2TLDWGeoMSM Operator}

When $m=2, p_{1}=1, p_{2}=2$, according to Section 4 , the procedures of the method are as follows:

Step 1. Normalizing the matrices 
Since values of each attribute are benefit-type, we have $R^{k}=\left[r_{i_{j}}^{k}\right]_{m \times n}=\left[x_{i_{j}}^{k}\right]_{m \times n}$.

Step 2. Converting linguistic decision information matrices $R^{k}=\left[r_{i_{j}}^{k}\right]_{m \times n}$ given in Tables 1-3 into matrices $R^{k}=\left[\left(r_{i_{j}}^{k}, 0\right)\right]_{m \times n}$ which are displayed in Tables 4-6.

Step 3. Aggregating all decision matrixes $(k=1,2,3)$ obtained in step 2 to the decision matrix $R=\left[\left(r_{i_{j}}, a_{i_{j}}\right)\right]_{4 \times 3}$ by 2TLDWGeoMSM, then following matrix can be obtained:

$$
R\left[\begin{array}{ccc}
\left(s_{1}, 0.2089\right) & \left(s_{1}, 0.3276\right) & \left(s_{1},-0.2523\right) \\
\left(s_{2},-0.4313\right) & \left(s_{1}, 0.3383\right) & \left(s_{1}, 0.2881\right) \\
\left(s_{1}, 0.2108\right) & \left(s_{1}, 0.2209\right) & \left(s_{1},-0.2253\right) \\
\left(s_{2},-4415\right) & \left(s_{1}, 0.2383\right) & \left(s_{1}, 0.3122\right)
\end{array}\right]
$$

Step 4. Calculating each alternative's comprehensive evaluation value $r_{i}=\left(r_{i}, a_{i}\right)$ by 2TLDWGeoMSM, we would get following result:

$$
r_{1}=\left(s_{1},-0.2179\right), r_{2}=\left(s_{1},-0.1622\right), r_{3}=\left(s_{1},-0.2235\right), r_{4}=\left(s_{1},-0.168\right)
$$

Step 5. Ranking the 2-tuple $r_{i}=\left(r_{i}, a_{i}\right)(i=1,2,3,4)$, the following sorted result can be obtained:

$$
r_{2}>r_{4}>r_{1}>r_{3}
$$

Step 6. Ranking all the alternatives $A=\left\{A_{1}, A_{2}, A_{3}, A_{4}\right\}$ in conformity to $r_{i}$, the sorted results are shown below:

$$
A_{2}>A_{4}>A_{1}>A_{3}
$$

Thus, the best one is $A_{2}$.

\subsection{Comparative Analysis and Discussion}

(1) According to the consequences in Sections 5.2-5.4 revealed in Table 7, we first compare our proposed three methods with two methods proposed by Liu [27] and Yager [34]. This comparison is revealed in Table 8. As we have seen in Table 8, same ordering of the alternatives would be produced by using 2TLDWGMSM operator, 2TLDWGeoMSM operator and 2TLBM operator in [34]. However, using 2TLDWMSM operator and 2TLHM operators in [27], another ordering would be obtained. It can be interpreted, both the Bonferroni mean (BM) operator and the MSM operator can capture the interrelationship between input arguments. Although the $\mathrm{HM}$ operator also takes the relationships between each attribute into account, it would ignore the correlations between the $C_{i}$ and $C_{j}$ when $i$ is less than $j$. Therefore, the comparisons in Tables 7 and 8 indicated that the developed MAGDM methods in this paper are useful and valid.

Table 7. Aggregation results.

\begin{tabular}{ccccc}
\hline Proposed Operator & $\boldsymbol{m}$ & $\boldsymbol{p}_{\mathbf{1}}$ & $\boldsymbol{p}_{\mathbf{2}}$ & Ranking \\
\hline $2 T L D W M S M^{(m)}$ & 2 & - & - & $A_{2}>A_{4}>A_{3}>A_{1}$ \\
$2 T L D W G M S M^{\left(m, p_{1}, p_{2}\right)}$ & 2 & 1 & 2 & $A_{2}>A_{4}>A_{1}>A_{3}$ \\
$2 T L D W G_{e 0} M S M^{\left(m, p_{1}, p_{2}\right)}$ & 2 & 1 & 2 & $A_{2}>A_{4}>A_{1}>A_{3}$ \\
\hline
\end{tabular}


Table 8. Comparison of different methods.

\begin{tabular}{ccc}
\hline Methods & Operator & Ranking \\
\hline Methods in this paper & $2 T L D W_{M S M}^{(m)} m=2$ & $A_{2}>A_{4}>A_{3}>A_{1}$ \\
Methods in this paper & $2 T L D W G M S M^{\left(m, p_{1}, p_{2}\right)} p_{1}=1 p_{2}=2$ & $A_{2}>A_{4}>A_{1}>A_{3}$ \\
Methods in this paper & $2 T L D W G_{e o} M^{\left(m, p_{1}, p_{2}\right)} p_{1}=1 p_{2}=2$ & $A_{2}>A_{4}>A_{1}>A_{3}$ \\
Method in [27] & $2 T L H M^{\left(m, p_{1}, p_{2}\right)} p_{1}=1 p_{2}=2$ & $A_{2}>A_{4}>A_{3}>A_{1}$ \\
Method in [34] & $2 T L B M^{\left(m, p_{1}, p_{2}\right)} p_{1}=1 p_{2}=2$ & $A_{2}>A_{4}>A_{1}>A_{3}$ \\
\hline
\end{tabular}

(2) Furthermore, a comparison of results is conducted to discuss the impact of the choice of parameters on the ordering.

Firstly, there are the comparisons for 2TLDWGMSM operator and 2TLDWGeoMSM operator with different values of $p_{1}$ and $p_{2}$ when $m=2$, which are displayed in Table 9. The data in Table 9 indicate the best option is $A_{2}$ and the worst option is $A_{1}$ or $A_{3}$ when the operator is selected. With respect to Table 9, we can discover that when $m=2$, we can get the same ranking results. Except when $p_{1}=p_{2}=0.5$, the result of ranking by 2TLDWGMSM is the same as the 2TLDWMSM when $m=2$. Therefore, the results in Table 9 prove the stability of the proposed method.

Table 9. Comparison results when $m=2$.

\begin{tabular}{|c|c|c|c|}
\hline$p_{1}$ & $p_{2}$ & Ranking by $2 T L D W G M S M^{\left(m, p_{1}, p_{2}\right)}$ & Ranking by $2 T L D W G_{e o} M S M^{\left(m, p_{1}, p_{2}\right)}$ \\
\hline 0.5 & 0.5 & $A_{2}>A_{4}>A_{3}>A_{1}$ & $A_{2}>A_{4}>A_{1}>A_{3}$ \\
\hline 1 & 0 & $A_{2}>A_{4}>A_{1}>A_{3}$ & $A_{2}>A_{4}>A_{1}>A_{3}$ \\
\hline 0 & 1 & $A_{2}>A_{4}>A_{1}>A_{3}$ & $A_{2}>A_{4}>A_{1}>A_{3}$ \\
\hline 1 & 2 & $A_{2}>A_{4}>A_{1}>A_{3}$ & $A_{2}>A_{4}>A_{1}>A_{3}$ \\
\hline 1 & 3 & $A_{2}>A_{4}>A_{1}>A_{3}$ & $A_{2}>A_{4}>A_{1}>A_{3}$ \\
\hline 2 & 1 & $A_{2}>A_{4}>A_{1}>A_{3}$ & $A_{2}>A_{4}>A_{1}>A_{3}$ \\
\hline 2 & 2 & $A_{2}>A_{4}>A_{1}>A_{3}$ & $A_{2}>A_{4}>A_{1}>A_{3}$ \\
\hline 2 & 3 & $A_{2}>A_{4}>A_{1}>A_{3}$ & $A_{2}>A_{4}>A_{1}>A_{3}$ \\
\hline 3 & 1 & $A_{2}>A_{4}>A_{1}>A_{3}$ & $A_{2}>A_{4}>A_{1}>A_{3}$ \\
\hline 3 & 2 & $A_{2}>A_{4}>A_{1}>A_{3}$ & $A_{2}>A_{4}>A_{1}>A_{3}$ \\
\hline 3 & 3 & $A_{2}>A_{4}>A_{1}>A_{3}$ & $A_{2}>A_{4}>A_{1}>A_{3}$ \\
\hline 4 & 4 & $A_{2}>A_{4}>A_{1}>A_{3}$ & $A_{2}>A_{4}>A_{1}>A_{3}$ \\
\hline 5 & 5 & $A_{2}>A_{4}>A_{1}>A_{3}$ & $A_{2}>A_{4}>A_{1}>A_{3}$ \\
\hline
\end{tabular}

Next, there are the comparisons for the 2TLDWGMSM operator and the 2TLDWGeoMSM operator with different values of $p_{1}, p_{2}$ and $p_{3}$ when $m=3$, which are displayed in Table 10 . When $p_{1}=0$ or $p_{2}=0$ or $p_{3}=0$, the impact of one of the attributes is not considered. So, the ranking results is different from others when $p_{1}, p_{2}$ and $p_{3}$ are not equal to zero. In addition, when the values of $p_{1}, p_{2}$, and $p_{3}$ are equal, we can get the ranking by 2TLDWGMSM is the same as the ranking by 2TLDWMSM. At the same time, the sorting results obtained by the 2TLDWGeoMSM are also the same. Furthermore, when two parameters are set the unchanged and equal value, the other parameter is incremented and not equal to the others, we can get two kinds of results based on the 2TLDWGMSM operator and the 2TLDWGeoMSM operator, the ordering in each class is the same. Therefore, the organizations can choose appropriate parameters and operators according to their interests and practical needs. 
Table 10. Comparison results when $m=2$.

\begin{tabular}{|c|c|c|c|c|}
\hline$p_{1}$ & $p_{2}$ & $p_{3}$ & Ranking by 2 TLDWGMSM ${ }^{\left(m, p_{1}, p_{2}\right)}$ & Ranking by $2 T L D W G_{e o} M S M^{\left(m, p_{1}, p_{2}\right)}$ \\
\hline 0.5 & 0.5 & 0.5 & $A_{2}>A_{4}>A_{3}>A_{1}$ & $A_{2}>A_{4}>A_{1}>A_{3}$ \\
\hline 1 & 1 & 0 & $A_{4}>A_{2}>A_{1}>A_{3}$ & $A_{4}>A_{2}>A_{1}>A_{3}$ \\
\hline 0 & 1 & 0 & $A_{2}>A_{4}>A_{1}>A_{3}$ & $A_{2}>A_{1}>A_{4}>A_{3}$ \\
\hline 0 & 0 & 1 & $A_{1}>A_{4}>A_{3}>A_{2}$ & $A_{1}>A_{3}>A_{4}>A_{2}$ \\
\hline 1 & 1 & 2 & $A_{2}>A_{4}>A_{1}>A_{3}$ & $A_{1}>A_{4}>A_{2}>A_{3}$ \\
\hline 1 & 1 & 3 & $A_{4}>A_{2}>A_{1}>A_{3}$ & $A_{1}>A_{4}>A_{2}>A_{3}$ \\
\hline 1 & 1 & 4 & $A_{4}>A_{1}>A_{2}>A_{3}$ & $A_{1}>A_{4}>A_{3}>A_{2}$ \\
\hline 1 & 1 & 5 & $A_{4}>A_{1}>A_{2}>A_{3}$ & $A_{1}>A_{3}>A_{4}>A_{2}$ \\
\hline 1 & 2 & 1 & $A_{2}>A_{3}>A_{1}>A_{4}$ & $A_{2}>A_{4}>A_{1}>A_{3}$ \\
\hline 1 & 3 & 1 & $A_{2}>A_{1}>A_{3}>A_{4}$ & $A_{2}>A_{1}>A_{3}>A_{4}$ \\
\hline 1 & 4 & 1 & $A_{2}>A_{4}>A_{1}>A_{3}$ & $A_{2}>A_{1}>A_{4}>A_{3}$ \\
\hline 1 & 5 & 1 & $A_{2}>A_{4}>A_{1}>A_{3}$ & $A_{2}>A_{1}>A_{4}>A_{3}$ \\
\hline 2 & 1 & 1 & $A_{4}>A_{2}>A_{1}>A_{3}$ & $A_{4}>A_{3}>A_{2}>A_{1}$ \\
\hline 3 & 1 & 1 & $A_{4}>A_{2}>A_{1}>A_{3}$ & $A_{4}>A_{3}>A_{2}>A_{1}$ \\
\hline 4 & 1 & 1 & $A_{4}>A_{2}>A_{1}>A_{3}$ & $A_{4}>A_{3}>A_{2}>A_{1}$ \\
\hline 5 & 1 & 1 & $A_{4}>A_{2}>A_{1}>A_{3}$ & $A_{2}>A_{4}>A_{3}>A_{1}$ \\
\hline 1 & 1 & 1 & $A_{2}>A_{4}>A_{3}>A_{1}$ & $A_{2}>A_{4}>A_{1}>A_{3}$ \\
\hline 2 & 2 & 2 & $A_{2}>A_{4}>A_{3}>A_{1}$ & $A_{2}>A_{4}>A_{1}>A_{3}$ \\
\hline 3 & 3 & 3 & $A_{2}>A_{4}>A_{3}>A_{1}$ & $A_{2}>A_{4}>A_{3}>A_{1}$ \\
\hline 4 & 4 & 4 & $A_{2}>A_{4}>A_{3}>A_{1}$ & $A_{2}>A_{4}>A_{3}>A_{1}$ \\
\hline
\end{tabular}

(3) Compared with other similar methods in a real scenario. Aiming at the problem of the large amount of information in modern multi-criterion group decision-making environment, Morente-Molinera et al. [35,36] proposed a method of using fuzzy ontologies: Experts determine the importance of each criteria and use fuzzy ontology to calculate the alternatives ranking automatically. In another method, Bagga et al. [37] calculated a Spearman's Rank Correlation Coefficient for different MCDM methods to show the least deviation in the MCDM methods. Shang [38] investigated resilient multiscale coordination algorithms which could withstand the compromise of a subset of nodes in directed networks. Furthermore, Shang [39] demonstrated how to provide resilience against such non-cooperative behaviors in opinion dynamics and established varied necessary and sufficient conditions for the hybrid opinion network to reach consensus in mean in the presence of globally and locally bounded non-rational agents based on a filtering strategy which removes some fixed number of opinion values. Unlike above methods, the method proposed in this paper presents a new solution from the aspect of decision operators. In this paper, the computational objects of operators take the form of 2-tuple, that is more comfortable for decision-maker when they provide the required information. In addition, the operators proposed in the paper can not only emphasize the relationship between any two attributes but also mitigate the unequitable parameters' impact on the overall outcome.

From the above comparison, it is important to select approximate parameters in the processes of solving the problem. In addition, it is obvious that methods proposed in this paper are advisable and feasible for MAGDM. Furthermore, they are more reliable than other existing methods.

\section{Conclusions}

In this paper, in order to deal with the MAGDM problems in which the attribute values are in 2-tuple linguistic information, some new operators and methods are studied. For the sake of solving the problem well, we proposed three operators based on dependent aggregation operators and MSM operators: The 2TLDWMSM, 2TLDWGMSM and 2TLDWGeoMSM operator, respectively. Different from other operators, MSM operators clarify the correlation among multiple input parameter by multiplication between $\Delta^{-1}\left(s_{i_{j}}, a_{i_{j}}\right)$ and $\Delta^{-1}\left(s_{i_{k}}, a_{i_{k}}\right)(j \neq k)$ in the formula when $i=1,2, \ldots, n$. Beyond that, the common of the above operators are the weights of each attribute value merely rely on the integrated 2-tuple linguistic parameters and they could mitigate the unequitable parameters' impact on the overall outcome in which those "wrong" and "prejudiced" parameters are distributed with low 
weights. Then a method for the MAGDM with 2-tuples linguistic information based on developed operators are developed. In the end, there is an illustrative instance to describe the procedures of the studied methods.

In the future, we would continue investigating and use our developed operators to address some practical decision-making problems, such as business investment and environmental evaluation and so on. In addition, in some special cases, 2-tuple linguistic information is not enough to express the fuzziness of human thought and the uncertainty of objects. Therefore, with respect to future research, we would discuss the effect of different operational laws on the final sort and extend our research from the MAGDM in 2-tuple linguistic information to picture fuzzy sets.

Author Contributions: In this article, a short paragraph specifying authors' individual contributions must be provided. Y.G. conceived and designed the experiments; M.F. analyzed the data; P.L. contributed analysis methods; M.F. wrote the paper.

Funding: This research was funded by Key R \& D project of Shandong Province, China, grant number is 2017 XCGC0605.

Conflicts of Interest: The authors declare no conflict of interest.

\section{References}

1. Zadeh, L.A. The concept of a linguistic variable and its application approximate reasoning, Part 1, 2, and Part 3. Inf. Sci. 1975, 8, 199-249. [CrossRef]

2. Yu, S.M.; Wang, J.; Wang, J.Q. An extended TODIM approach with intuitionistic linguistic numbers. Int. Trans. Oper. Res. 2016, 25, 781-805. [CrossRef]

3. Wang, J.; Wang, J.Q.; Zhang, H.Y. A likelihood-based TODIM approach based on multi-hesitant fuzzy linguistic information for evaluation in logistics outsourcing. Comput. Ind. Eng. 2016, 99, 287-299. [CrossRef]

4. Moharrer, M.; Tahayori, H.; Livi, L. Interval type-2 fuzzy sets to model linguistic label perception in online services satisfaction. Soft Comp. 2015, 19, 237-250. [CrossRef]

5. Herrera, F.; Herrera-Viedma, E.; Verdegay, J.L. A sequential selection process in group decision making with linguistic assessment. Inf. Sci. 1995, 85, 223-239. [CrossRef]

6. Herrera, F.; Herrera-Viedma, E.; Verdegay, J.L. Direct approach processes in group decision making using linguistic OWA operators. Fuzzy Sets Syst. 1996, 79, 175-190. [CrossRef]

7. $\mathrm{Xu}, \mathrm{Z}$.S. A method based on linguistic aggregation operators for group decision making with linguistic preference relations. Inf. Sci. 2004, 166, 19-30. [CrossRef]

8. Herrera, F.; Martinez, L. A 2-tuple fuzzy linguistic representation model for computing with words. IEEE Trans. Fuzzy Syst. 2000, 8, 746-752.

9. Jiang, Y.P.; Fan, Z.P. Property analysis of the aggregation operators for 2-tuple linguistic information. Control Decis. 2003, 18, 754-757.

10. Wei, G.W. Some harmonic aggregation operators with 2-tuple linguistic assessment information and their application to multiple attribute group decision making. Int. J. Uncertain. Fuzz. Knowl. Based Syst. 2011, 19, 977-998. [CrossRef]

11. Merigo, J.M.; Gil-Lafuente, A.M. Induced 2-tuple linguistic generalized aggregation operators and their application in decision-making. Inf. Sci. 2013, 226, 1-16. [CrossRef]

12. Wang, J.; Wang, J.Q.; Zhang, H.Y.; Chen, X.H. Multi-criteria group decision-making approach based on 2-tuple linguistic aggregation operators with multi-hesitant fuzzy linguistic information. Int. J. Fuzzy Syst. 2015. [CrossRef]

13. Deng, X.; Wang, J.; Wei, G.; Lu, M. Models for Multiple Attribute Decision Making with Some 2-Tuple Linguistic Pythagorean Fuzzy Hamy Mean Operators. Mathematics 2018, 6, 236. [CrossRef]

14. Maclaurin, C. A second letter to Martin Folkes, Esq.: Concerning the roots of equations, with the demonstration of other rules of algebra. Philos. Trans. R. Soc. Lond. Ser. A 1972, 36, 59-96.

15. Detemple, D.; Robertson, J. On generalized symmetric means of two variables. Angew. Chem. 1979, 47, 4638-4660.

16. Qin, J.; Liu, X. An Approach to Intuitionistic Fuzzy Multiple Attribute Decision Making Based on Maclaurin Symmetric Mean Operators; IOS Press: Amsterdam, The Netherlands, 2014. 
17. Qin, J.; Liu, X.; Pedrycz, W. Hesitant Fuzzy Maclaurin Symmetric Mean Operators and Its Application to Multiple-Attribute Decision Making. Int. J. Fuzzy Syst. 2015, 17, 509-520. [CrossRef]

18. Wang, J.Q.; Yang, Y.; Li, L. Multi-criteria decision-making method based on single-valued neutrosophic linguistic Maclaurin symmetric mean operators. Neural Comput. Appl. 2016, 4, 1-19. [CrossRef]

19. Geng, Y.S.; Wang, X.G.; Li, X.M.; Yu, K. Some Interval Neutrosophic Linguistic Maclaurin Symmetric Mean Operators and their Application in Multiple Attribute Decision Making. Symmetry 2018, 10, 127. [CrossRef]

20. Wang, J.; Wei, G.; Gao, H. Approaches to Multiple Attribute Decision Making with Interval-Valued 2-Tuple Linguistic Pythagorean Fuzzy Information. Mathematics 2018, 6, 201. [CrossRef]

21. Xu, Z. Dependent uncertain ordered weighted aggregation operators. Inf. Fusion 2008, 9, 310-316. [CrossRef]

22. Wei, G.W.; Zhao, X.F. Some dependent aggregation operators with 2-tuple linguistic information and their application to multiple attribute group decision making. Expert Syst. Appl. 2012, 39, 5881-5886. [CrossRef]

23. Liu, P.; Liu, P.D. Some generalized dependent aggregation operators with intuitionistic linguistic numbers and their application to group decision making. J. Comput. Syst. Sci. 2013, 79, 131-143. [CrossRef]

24. Gao, J.; Liu, H. Reference-Dependent Aggregation in Multi-AttributeGroup Decision-Making. Symmetry 2017, 9, 43. [CrossRef]

25. Herrera, F.; Herrera-Viedma, E.; Verdegay, J.L. A model of consensus in group decision making under linguistic assessments. Fuzzy Sets Syst. 1996, 79, 73-87. [CrossRef]

26. Herrera, F.; Herrera-Viedma, E. Linguistic Decision Analysis: Steps for Solving Decision Problems Under Linguistic Information. Fuzzy Sets Syst. 2000, 115, 67-82. [CrossRef]

27. Liu, P.D. Some Heronian mean operators with 2-tuple linguistic information and their application to multiple attribute group decision making. Technol. Econ. Dev. Econ. 2015, 21, 797-814.

28. Xu, Z.S. A note on linguistic hybrid arithmetic averaging operator in multiple attribute group decision making with linguistic information. Group Decis. Negot. 2006, 15, 593-604. [CrossRef]

29. Xu, Z.S. Goal programming models for multiple attribute decision making under linguistic setting. J. Manag. Sci. China 2006, 9, 9-17.

30. Herrera, F.; Martinez, L.; Sanchez, P.J. Managing non-Homogeneous information in group decision making. Eur. J. Oper. Res. 2005, 166, 115-132. [CrossRef]

31. Zhang, Z.H.; Wei, F.; Zhou, S. Approaches to Comprehensive Evaluation with 2-Tuple Linguistic Information; IOS Press: Amsterdam, The Netherlands, 2015.

32. Shang, Y. Finite-Time Weighted Average Consensus and Generalized Consensus Over a Subset. IEEE Access 2016, 4, 2615-2620. [CrossRef]

33. Xu, Y.J.; Wang, H.M. Approaches based on 2-tuple linguistic power aggregation operators for multiple attribute group decision making under linguistic environment. Appl. Soft Comput. 2011, 11, 3988-3997. [CrossRef]

34. Yager, R.R. On generalized Bonferroni mean operators for multi-criteria aggregation. Int. J. Approx. Reason. 2009, 50, 1279-1286. [CrossRef]

35. Managing Multi-Criteria Group Decision Making Environments With High Number Of Alternatives Using Fuzzy Ontologies. Available online: https://www.researchgate.net/publication/325934668_Managing_ multi-criteria_group_decision_making_environments_with_high_number_of_alternatives_using_fuzzy_ ontologies (accessed on 2 December 2018).

36. Morente-Molineraa, J.A.; Kou, G.; González-Crespo, R.; Corchado, J.M.; Herrera-Viedma, E. Solving multi-criteria group decision making problems under environments with a high number of alternatives using fuzzy ontologies and multi-granular linguistic modelling methods. Knowl. Based Syst. 2017, 13, 54-64. [CrossRef]

37. Bagga, P.; Joshi, A.; Hans, R. QoS based Web Service Selection and Multi-Criteria Decision Making Methods. Int. J. Interact. Multimed. Artif. Intell. 2017. [CrossRef]

38. Shang, Y.L. Resilient Multiscale Coordination Control against Adversarial Nodes. Energies 2018, $11,1844$. [CrossRef]

39. Shang, Y.L. Hybrid consensus for averager-copier-voter networks with non-rational agents. Chaos Solitons Fractals 2018, 110, 244-251. [CrossRef]

(C) 2019 by the authors. Licensee MDPI, Basel, Switzerland. This article is an open access article distributed under the terms and conditions of the Creative Commons Attribution (CC BY) license (http:/ / creativecommons.org/licenses/by/4.0/). 